Supporting information

\title{
Radical Trifluoromethylation of Ketone Silyl Enol Ethers by Activation with Dialkylzinc
}

Koichi Mikami, * Yuichi Tomita, Yoshiyuki Ichikawa, Kazutoshi Amikura, and Yoshimitsu Itoh

\section{General :}

${ }^{1} \mathrm{H}$ NMR and ${ }^{13} \mathrm{C}$ NMR were measured on Varian Gemini 2000 (300 MHz) spectrometers and ${ }^{19} \mathrm{~F}$ NMR was measured on Varian UNITY INOVA (400 MHz) spectrometers. Chemical shift of ${ }^{1} \mathrm{H}$ NMR was expressed in parts per million downfield from tetramethylsilane as an internal standard $(\delta=0)$ in $\mathrm{CDCl}_{3}$. Chemical shifts of ${ }^{13} \mathrm{C}$ NMR were expressed in parts per million downfield from $\mathrm{CDCl}_{3}$ as an internal standard $(\delta=77.0)$ in $\mathrm{CDCl}_{3}$. Chemical shifts of ${ }^{19} \mathrm{~F}$ NMR were expressed in parts per million downfield from BTF as an internal standard $(\delta=-63.24)$ in $\mathrm{CDCl}_{3}$. IR spectrum was measured on JASCO FT/IR-5000 spectrometer. EI Mass spectra were measured on JEOL JMS-700 spectrometer. Analytical thin layer chromatographies (TLC) were performed on a glass plates and /or aluminum sheets pre-coated with silica gel (Merck Kieselgal $60 \mathrm{~F}_{254}$, layer thickness 0.25 and $0.2 \mathrm{~mm}$ ). Visualization was accomplished by UV light $(254 \mathrm{~nm})$, anisaldehyde, $\mathrm{KMnO}_{4}$ and phosphomolybdic acid. Column chromatography was performed on Merck Kieselgel 60 and KANTO Silica Gel 60N (spherical, neutral), employing hexane and dichloromethane mixture as an eluent unless otherwise noted. THF was distilled from benzophenone-kethyl under Ar prior to use. All experiments were carried out under argon atmosphere unless otherwise noted.

\section{Typical experimental procedure of trifluoromethylation of silyl enol} ethers by activation with dialkylzinc

To a solution of 1 -(trimethylsilyloxy) cyclohexene $(38.9 \mu \mathrm{L}, 0.2 \mathrm{mmol}$ ) in $\mathrm{THF}$ was added $\mathrm{Et}_{2} \mathrm{Zn}(200 \mu \mathrm{L}$ of $1.0 \mathrm{M}$ solution in hexane, $0.2 \mathrm{mmol})$ at $0{ }^{\circ} \mathrm{C}$ and stirred for 1 hour at the temperature. Then, the reaction mixture was cooled to $-78{ }^{\circ} \mathrm{C}$. To the mixture was added gaseous $\mathrm{CF}_{3} \mathrm{I}$ ( $\mathrm{Ca}$. $200 \mathrm{mg}$, ca. $1.0 \mathrm{mmol})$ with a cannula followed by $E t_{3} B(0.2 \mathrm{~mL}$ of 1.0 $\mathrm{M}$ solution in hexane, $0.2 \mathrm{mmol})$. And then air $(0.1 \mathrm{~mL})$ was added with syringe. The reaction mixture was stirred for 1 hour at $-78{ }^{\circ} \mathrm{C}$ and then quenched by acetic acid ( $5 \mathrm{M}$ solution in THF) at $-78{ }^{\circ} \mathrm{C}$. After warming to room temperature, BTF $(10 \mu \mathrm{L}, 0.082 \mathrm{mmol})$ was added as an internal standard. The yield was determined by ${ }^{19} \mathrm{~F}$ NMR of the crude mixture (75\%).

2-Trifluoromethyl-cyclohexanone $(2 a)^{1,2}$

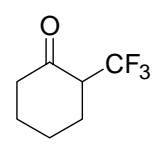

${ }^{1} \mathrm{H} \quad \mathrm{NMR}\left(\mathrm{CDCl}_{3}, 400 \mathrm{MHz}\right)$

$\delta 1.62 \sim 1.88(\mathrm{~m}, 3 \mathrm{H}), 1.92 \sim 2.14(\mathrm{~m}, 2 \mathrm{H}), 2.24 \sim 2.39(\mathrm{~m}, 2 \mathrm{H}), 2.42 \sim 2.53$ $(\mathrm{m}, 1 \mathrm{H}), 2.98 \sim 3.13(\mathrm{~m}, 1 \mathrm{H})(\mathrm{ppm})$.

${ }^{13} \mathrm{C}$ NMR $\left(\mathrm{CDCl}_{3}, 75 \mathrm{~Hz}\right)$

$\delta 23.7,27.1,27.5(q, J=2.4 \mathrm{~Hz}), 42.2,53.6(q, J=25.7 \mathrm{~Hz}), 124.6(q$, 
$J=279.5 \mathrm{~Hz}), 203.0$ (ppm) .

${ }^{19} \mathrm{~F}$ NMR $\left(\mathrm{CDCl}_{3}, 376 \mathrm{~Hz}\right)$

$\delta-69.3(\mathrm{~d}, \mathrm{~J}=7.9 \mathrm{~Hz})$ (ppm) .

IR (neat)

$2954,2876,2364,1729,1272,1170,1125,1060\left(\mathrm{~cm}^{-1}\right)$

HRMS (EI)

Calcd for $\mathrm{C}_{7} \mathrm{H}_{9} \mathrm{~F}_{3} \mathrm{O}\left[\mathrm{M}^{+\cdot}\right]$ : 166.0605, Found: 166.0609 .

2-Metyl-2-trifluoromethyl-cyclohexanone (2b) $)^{2,3}$

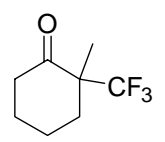

${ }^{1} \mathrm{H} \quad \mathrm{NMR}\left(\mathrm{CDCl}_{3}, 300 \mathrm{MHz}\right)$

$\delta 1.36(\mathrm{~s}, 3 \mathrm{H}), 1.70 \sim 2.00(\mathrm{~m}, 5 \mathrm{H}), 2.06 \sim 2.20(\mathrm{~m}, 1 \mathrm{H}), 2.35 \sim 2.58(\mathrm{~m}$, 2H) (ppm) .

${ }^{13} \mathrm{C}$ NMR $\left(\mathrm{CDCl}_{3}, 75 \mathrm{~Hz}\right)$

$\delta 17.7(q, J=2.4 \mathrm{~Hz}), 20.5,26.4,33.5,39.4,53.7(q, J=23.2 \mathrm{~Hz}), 126.5$ (q, J=283.2 Hz), 206.2 (ppm).

${ }^{19} \mathrm{~F}$ NMR $\left(\mathrm{CDCl}_{3}, 376 \mathrm{~Hz}\right)$

$\delta-73.6$ (s) (ppm) .

IR (neat)

2936, 2874, 1725, 1274, 1170, $1137\left(\mathrm{~cm}^{-1}\right)$

HRMS (EI)

Calcd for $\mathrm{C}_{8} \mathrm{H}_{11} \mathrm{~F}_{3} \mathrm{O}\left[\mathrm{M}^{+\bullet}\right]: 180.0762$, Found: 180.0760 .

2-Methyl-6-trifluoromethyl-cyclohexanone $(2 \mathrm{c})^{2,3}$

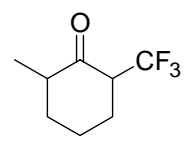

trans isomer

${ }^{1} \mathrm{H}$ NMR $\left(\mathrm{CDCl}_{3}, 300 \mathrm{MHz}\right)$

$\delta 1.11(\mathrm{~d}, \mathrm{~J}=6.6 \mathrm{~Hz}, 3 \mathrm{H}), 1.46 \sim 2.21(\mathrm{~m}, 6 \mathrm{H}), 2.57 \sim 2.71(\mathrm{~m}, 1 \mathrm{H}), 3.07 \sim 3.22$ $(\mathrm{m}, 1 \mathrm{H})(\mathrm{ppm})$.

${ }^{13} \mathrm{C}$ NMR $\left(\mathrm{CDCl}_{3}, 75 \mathrm{~Hz}\right)$

$\delta 15.0,20.2,26.9,29.6,34.2,44.5,52.3$ (q, J=25.7 Hz), 125.2 (q, $\mathrm{J}=280.7 \mathrm{~Hz}), 206.3$ (ppm) .

${ }^{19} \mathrm{~F} \quad \mathrm{NMR} \quad\left(\mathrm{CDCl}_{3}, 376 \mathrm{~Hz}\right)$

$\delta-66.7(\mathrm{~d}, J=10.2 \mathrm{~Hz})(\mathrm{ppm})$.

IR (neat)

$2928,2858,2364,2344,1725,1458,1265,1143,801\left(\mathrm{~cm}^{-1}\right)$

HRMS (EI)

Calcd for $\mathrm{C}_{7} \mathrm{H}_{9} \mathrm{~F}_{3} \mathrm{O}\left[\mathrm{M}^{+\bullet}\right]: 180.0762$, Found: 180.0766 .

Cis isomer

${ }^{1} \mathrm{H} \operatorname{NMR}\left(\mathrm{CDCl}_{3}, 300 \mathrm{MHz}\right)$

$\delta 1.03(\mathrm{~d}, J=6.3 \mathrm{~Hz}, 3 \mathrm{H}), 1.34 \sim 1.49(\mathrm{~m}, 1 \mathrm{H}), 1.63 \sim 1.87(\mathrm{~m}, 2 \mathrm{H}), 1.88 \sim 2.03$ $(\mathrm{m}, 1 \mathrm{H}), 2.08 \sim 2.19(\mathrm{~m}, 1 \mathrm{H}), 2.30 \sim 2.49(\mathrm{~m}, 2 \mathrm{H}), 2.98 \sim 3.16(\mathrm{~m}, 1 \mathrm{H})(\mathrm{ppm})$.

${ }^{13} \mathrm{C} \mathrm{NMR}\left(\mathrm{CDCl}_{3}, 75 \mathrm{~Hz}\right)$

$\delta 13.8,24.0,28.3,36.3,45.9,53.7(q, J=25.7 \mathrm{~Hz}), 124.8(q, J=279.5$ 
$\mathrm{Hz}), 204.6$ (ppm) .

${ }^{19} \mathrm{~F}$ NMR $\left(\mathrm{CDCl}_{3}, 376 \mathrm{~Hz}\right)$

$\delta \quad-69.8(\mathrm{~d}, \mathrm{~J}=8.3 \mathrm{~Hz})(\mathrm{ppm})$.

IR (neat)

2942, 2874, 2366, 1731, 1456, 1392, 1332, 1272, 1170, 1137, 1123, 1038, $832,688\left(\mathrm{~cm}^{-1}\right)$

HRMS (EI)

Calcd for $\mathrm{C}_{7} \mathrm{H}_{9} \mathrm{~F}_{3} \mathrm{O}\left[\mathrm{M}^{+\bullet}\right]: 180.0762$, Found: 180.0771 .

2-Phenyl-6-trifluoromethyl-cyclohexanone $(2 \mathrm{~d})^{2}$<smiles>O=C1C(c2ccccc2)CCCC1C(F)(F)F</smiles>

Major isomer

${ }^{1} \mathrm{H} \operatorname{NMR}\left(\mathrm{CDCl}_{3}, 300 \mathrm{MHz}\right)$

$\delta 1.86 \sim 2.28(\mathrm{~m}, 5 \mathrm{H}), 2.37 \sim 2.52(\mathrm{~m}, 1 \mathrm{H}), 3.12 \sim 3.30(\mathrm{dq}, J=6.0,9.3 \mathrm{~Hz}$, $1 \mathrm{H}), 3.82 \sim 3.92$ (distorted $t, J=6.3 \mathrm{~Hz}, 1 \mathrm{H}), 7.17 \sim 7.43(\mathrm{~m}, 5 \mathrm{H})$ (ppm) . ${ }^{13} \mathrm{C}$ NMR $\left(\mathrm{CDCl}_{3}, 75 \mathrm{~Hz}\right)$

$\delta 20.4,27.4,31.3,52.0(q, J=26.9 \mathrm{~Hz}), 55.1,125.1$ (q, J=280.8 Hz), $127.4,127.6,129.0,136.7,203.5$ (ppm).

${ }^{19} \mathrm{~F}$ NMR $\left(\mathrm{CDCl}_{3}, 376 \mathrm{~Hz}\right)$

$\delta-67.9(\mathrm{~d}, J=9.0 \mathrm{~Hz})$ (ppm) .

IR (neat)

$3066,3032,2954,2878,2364,1725,1603,1584,1499,1454,1390,1332$, $1274,1183,1141,698\left(\mathrm{~cm}^{-1}\right)$

HRMS (EI)

Calcd for $\mathrm{C}_{13} \mathrm{H}_{13} \mathrm{~F}_{3} \mathrm{O}\left[\mathrm{M}^{+\bullet}\right]: 242.0918$, Found: 242.0911.

Minor isomer

${ }^{1} \mathrm{H} \operatorname{NMR}\left(\mathrm{CDCl}_{3}, 300 \mathrm{MHz}\right)$

$\delta 1.83 \sim 2.21(\mathrm{~m}, 4 \mathrm{H}), 2.28 \sim 2.40(\mathrm{~m}, 1 \mathrm{H}), 2.40 \sim 2.56(\mathrm{~m}, 1 \mathrm{H}), 3.16 \sim 3.35$ $(\mathrm{m}, 1 \mathrm{H}), 3.56 \sim 3.68(\mathrm{dd}, J=5.4,12.6 \mathrm{~Hz}, 1 \mathrm{H}), 7.11 \sim 7.17(\mathrm{~m}, 2 \mathrm{H}), 7.25 \sim 7.40$ $(\mathrm{m}, 3 \mathrm{H})(\mathrm{ppm})$.

${ }^{13} \mathrm{C}$ NMR $\left(\mathrm{CDCl}_{3}, 75 \mathrm{~Hz}\right)$

$\delta 24.2,28.3,35.6,54.1$ (q, J=25.6 Hz), $57.8,124.6$ (q, J=280.8 Hz), $127.4,128.4,128.8,137.0,201.6$ (ppm).

${ }^{19} \mathrm{~F} \quad \mathrm{NMR}\left(\mathrm{CDCl}_{3}, 376 \mathrm{~Hz}\right)$

$\delta-69.6(\mathrm{~d}, J=7.9 \mathrm{~Hz})$ (ppm) .

IR $(\mathrm{KBr})$

$3036,2946,2872,1722,1605,1452,1385,1270,1168,1133,1045,761$, $704,592\left(\mathrm{~cm}^{-1}\right)$

HRMS (EI)

Calcd for $\mathrm{C}_{13} \mathrm{H}_{13} \mathrm{~F}_{3} \mathrm{O}\left[\mathrm{M}^{+\bullet}\right]$ : 242.0918, Found: 242.0909 .

2-Trifluoromethyl-cyclopentanone $(2 e)^{2,4}$

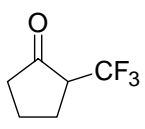

${ }^{1} \mathrm{H}$ NMR $\left(\mathrm{CDCl}_{3}, 300 \mathrm{MHz}\right)$ 
$\delta 1.77 \sim 2.00(\mathrm{~m}, 1 \mathrm{H}), 2.01 \sim 2.21(\mathrm{~m}, 2 \mathrm{H}), 2.22 \sim 2.48(\mathrm{~m}, 3 \mathrm{H}), 2.78 \sim 2.97$ ( qm, $J=9.6 \mathrm{~Hz}, 1 \mathrm{H}$ ) (ppm).

${ }^{13} \mathrm{C} \mathrm{NMR}\left(\mathrm{CDCl}_{3}, 75 \mathrm{~Hz}\right)$

$\delta 20.0,24.4,38.5,51.1(q, J=26.9 \mathrm{~Hz}), 124.6(q, J=278.3 \mathrm{~Hz}), 209.4$ (ppm).

${ }^{19} \mathrm{~F} \mathrm{NMR}\left(\mathrm{CDCl}_{3}, 376 \mathrm{~Hz}\right)$

$\delta \quad-67.9(\mathrm{~d}, J=10.5 \mathrm{~Hz})$ (ppm).

IR (neat)

$2986,2896,2366,2344,1758,1638,1367,1313,1257,1187,1151,1096$, $1046\left(\mathrm{~cm}^{-1}\right)$

HRMS (EI)

Calcd for $\mathrm{C}_{6} \mathrm{H}_{7} \mathrm{~F}_{3} \mathrm{O}\left[\mathrm{M}^{+\bullet}\right]: 152.0449$, Found: 152.0447 .

2-Trifluoromethyl-cycloheptanone $(2 f)^{2,5}$

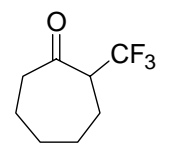

${ }^{1} \mathrm{H}$ NMR $\left(\mathrm{CDCl}_{3}, 300 \mathrm{MHz}\right)$

$\delta 1.22 \sim 1.48(\mathrm{~m}, 2 \mathrm{H}), 1.48 \sim 1.75(\mathrm{~m}, 2 \mathrm{H}), 1.86 \sim 2.05(\mathrm{~m}, 3 \mathrm{H}), 2.09 \sim 2.20$ $(\mathrm{m}, 1 \mathrm{H}), 2.54 \sim 2.61(\mathrm{~m}, 2 \mathrm{H}), 3.16 \sim 3.31$ (qdd, J=4.1, 8.9, $11.1 \mathrm{~Hz}, 1 \mathrm{H})$ (ppm) .

${ }^{13} \mathrm{C} \mathrm{NMR}\left(\mathrm{CDCl}_{3}, 75 \mathrm{~Hz}\right)$

$\delta 24.4,24.7(q, J=2.4 \mathrm{~Hz}), 27.5,29.1,43.1,55.5(q, J=24.5 \mathrm{~Hz}), 124.9$ (q, $J=280.8 \mathrm{~Hz}), 205.9$ (ppm).

${ }^{19} \mathrm{~F} \mathrm{NMR}\left(\mathrm{CDCl}_{3}, 376 \mathrm{~Hz}\right)$

$\delta-69.0(\mathrm{~d}, J=9.0 \mathrm{~Hz}) \quad(\mathrm{ppm})$.

IR (neat)

$2940,2866,1721,1178,1151,1096\left(\mathrm{~cm}^{-1}\right)$

HRMS (EI)

Calcd for $\mathrm{C}_{8} \mathrm{H}_{11} \mathrm{~F}_{3} \mathrm{O}\left[\mathrm{M}^{+\bullet}\right]: 180.0762$, Found: 180.0770 .

7-Trifluoromethyl-6-undecanone $(2 \mathrm{~g})^{2,6}$<smiles>CCCC(C(=O)NC)C(C(F)(F)F)C(F)(F)F</smiles>

${ }^{1} \mathrm{H} \mathrm{NMR}\left(\mathrm{CDCl}_{3}, 300 \mathrm{MHz}\right)$

$\delta 0.90(t, J=6.9 \mathrm{~Hz}, 6 \mathrm{H}), 1.18 \sim 1.41(\mathrm{~m}, 8 \mathrm{H}), 1.53 \sim 1.65(\mathrm{~m}, 2 \mathrm{H}), 1.65 \sim 1.79$ $(\mathrm{m}, 1 \mathrm{H}), 1.81 \sim 1.97(\mathrm{~m}, 1 \mathrm{H}), 2.47(\mathrm{dt}, \mathrm{J}=18.0,7.2 \mathrm{~Hz}, 1 \mathrm{H}), 2.61$ (dt, $\mathrm{J}=7.4,18.0 \mathrm{~Hz}, 1 \mathrm{H}), 3.11 \sim 3.26(\mathrm{~m}, 1 \mathrm{H})$ (ppm).

${ }^{13} \mathrm{C} \mathrm{NMR}\left(\mathrm{CDCl}_{3}, 75 \mathrm{~Hz}\right)$

$\delta 13.5,13.7,22.3,22.6,25.49,25.53,28.9,31.0,43.6,55.6(q, J=24.4$ $\mathrm{Hz}), 125.1$ (q, J=280.7 Hz), 204.7 (ppm).

${ }^{19} \mathrm{~F} \mathrm{NMR}\left(\mathrm{CDCl}_{3}, 376 \mathrm{~Hz}\right)$

$\delta \quad-67.4(\mathrm{~d}, J=9.0 \mathrm{~Hz})$ (ppm).

IR (neat)

$2966,2938,2870,1731,1263,1164\left(\mathrm{~cm}^{-1}\right)$

HRMS (EI)

Calcd for $\mathrm{C}_{12} \mathrm{H}_{21} \mathrm{~F}_{3} \mathrm{O}\left[\mathrm{M}^{+\bullet}\right]: 238.1544$, Found: 238.1540 . 

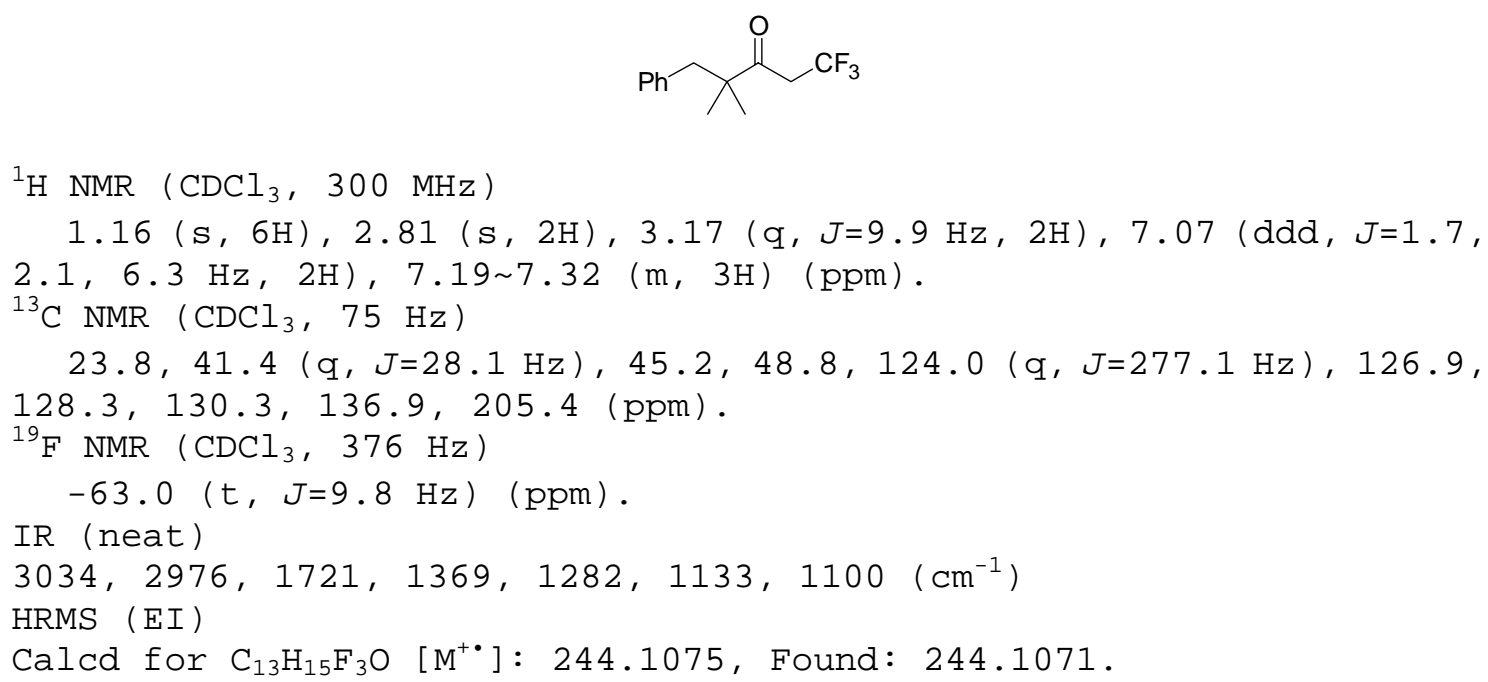

(1) (a) Cantacuzène, D.; Wakselman, C.; Dorme, R. J. Chem. Soc., Perkin Trans. 1 1977, 1365-1371.

(b) Tordeux, M.; Francese, C.; Walkselman, C. J. Chem. Soc., Perkin Trans. 1 1990, 1951-1957.

(2) (a) Itoh, Y.; Mikami, K. Org. Lett. 2005, 7, 649-651. (b) Itoh, Y.; Mikami, K. Org. Lett. 2005, 7, 4883-4885.

(3) Umemoto, T.; Adachi, K. J. Org. Chem. 1994, 59, 5692-5699.

(4) Semisch, C.; Margaretha, P. J. Fluorine. Chem. 1984, 30, 471-475.

(5) Mock, W. L.; Hartman, M. E. J. Org. Chem. 1977, 42, 459-465.

(6) Miura, K.; Takeyama, Y.; Oshima, K.; Utimoto, K. Bull. Chem. Soc. Jpn. 1991, 64, 1542-1553. 

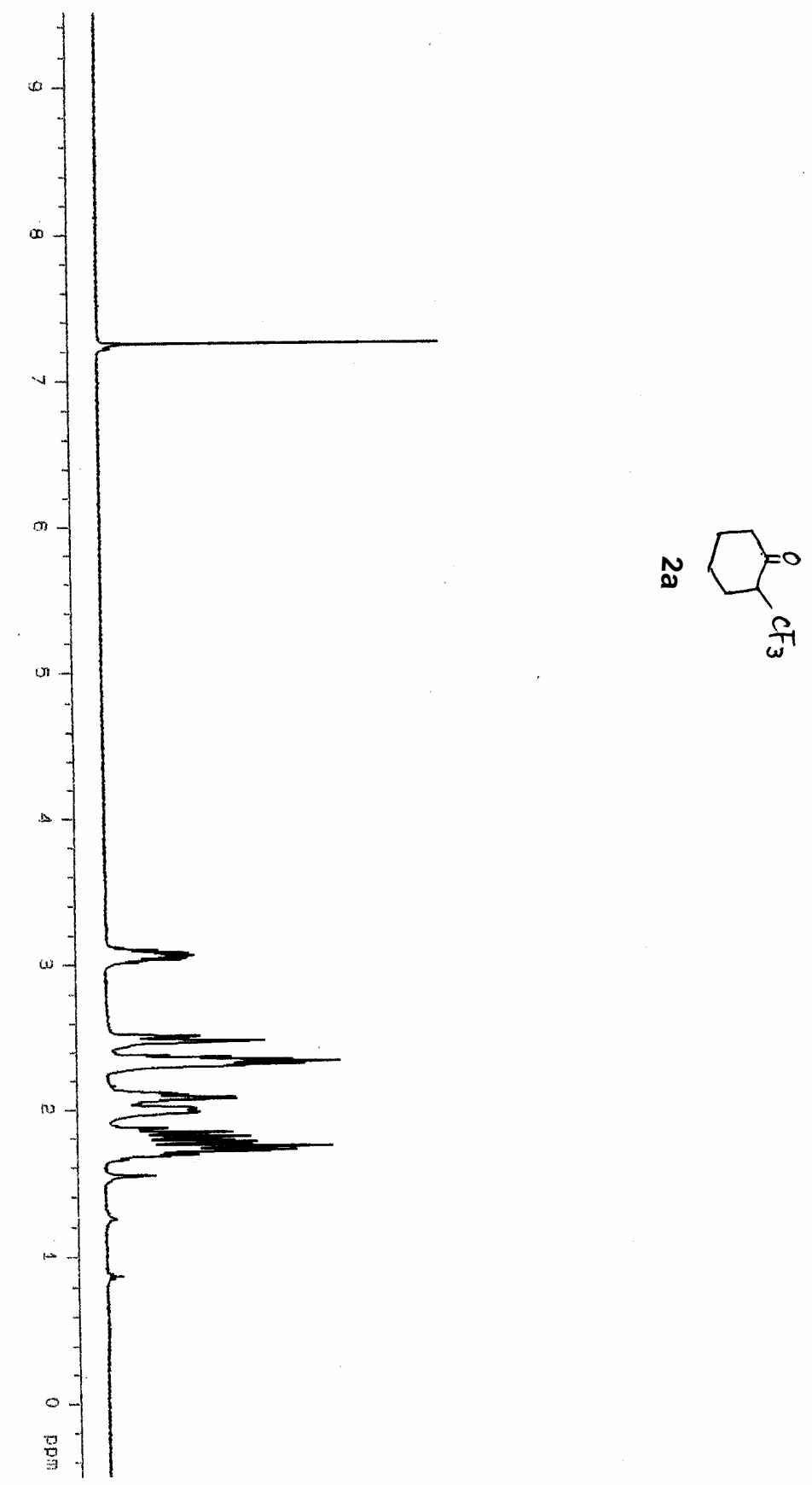

S6 


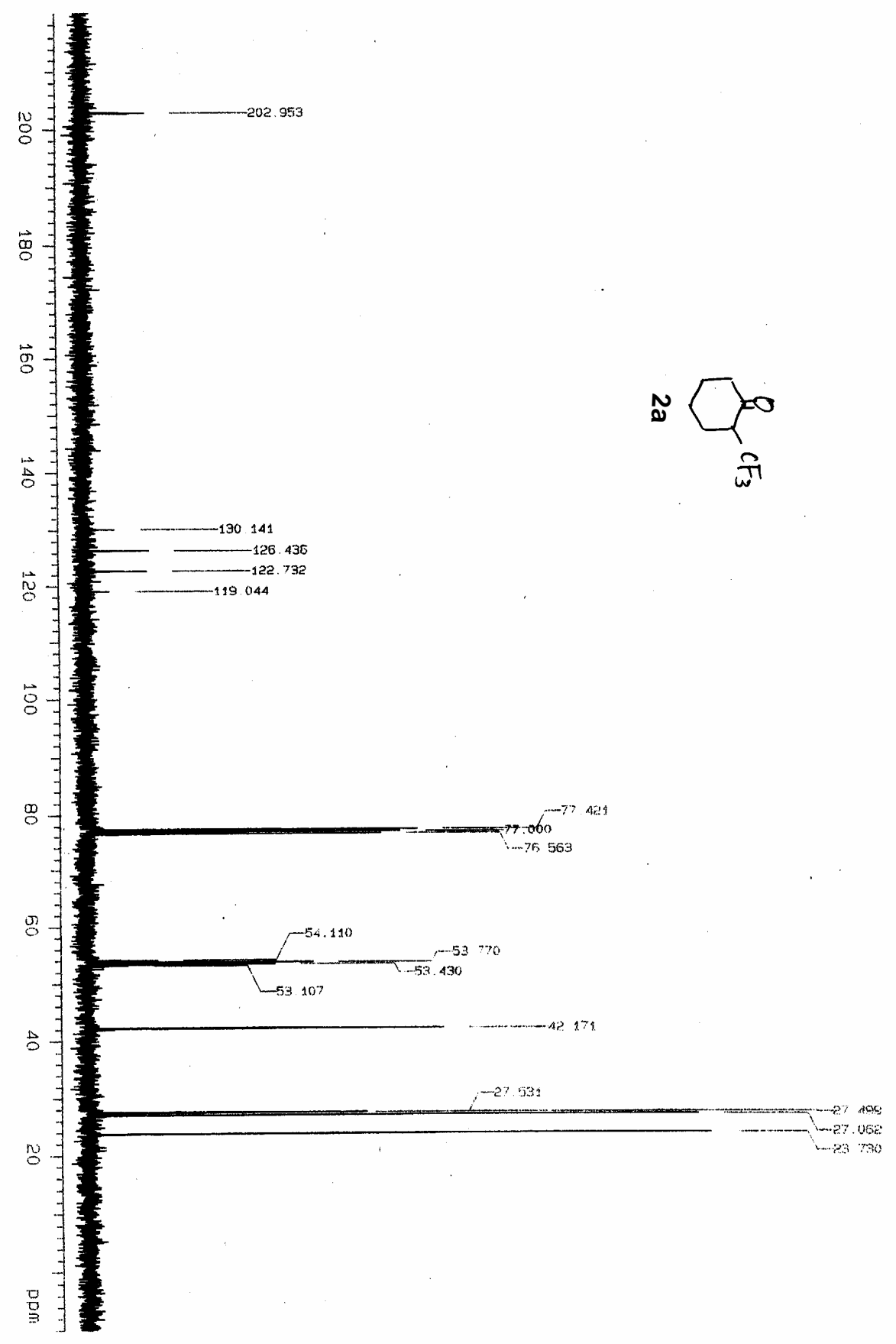




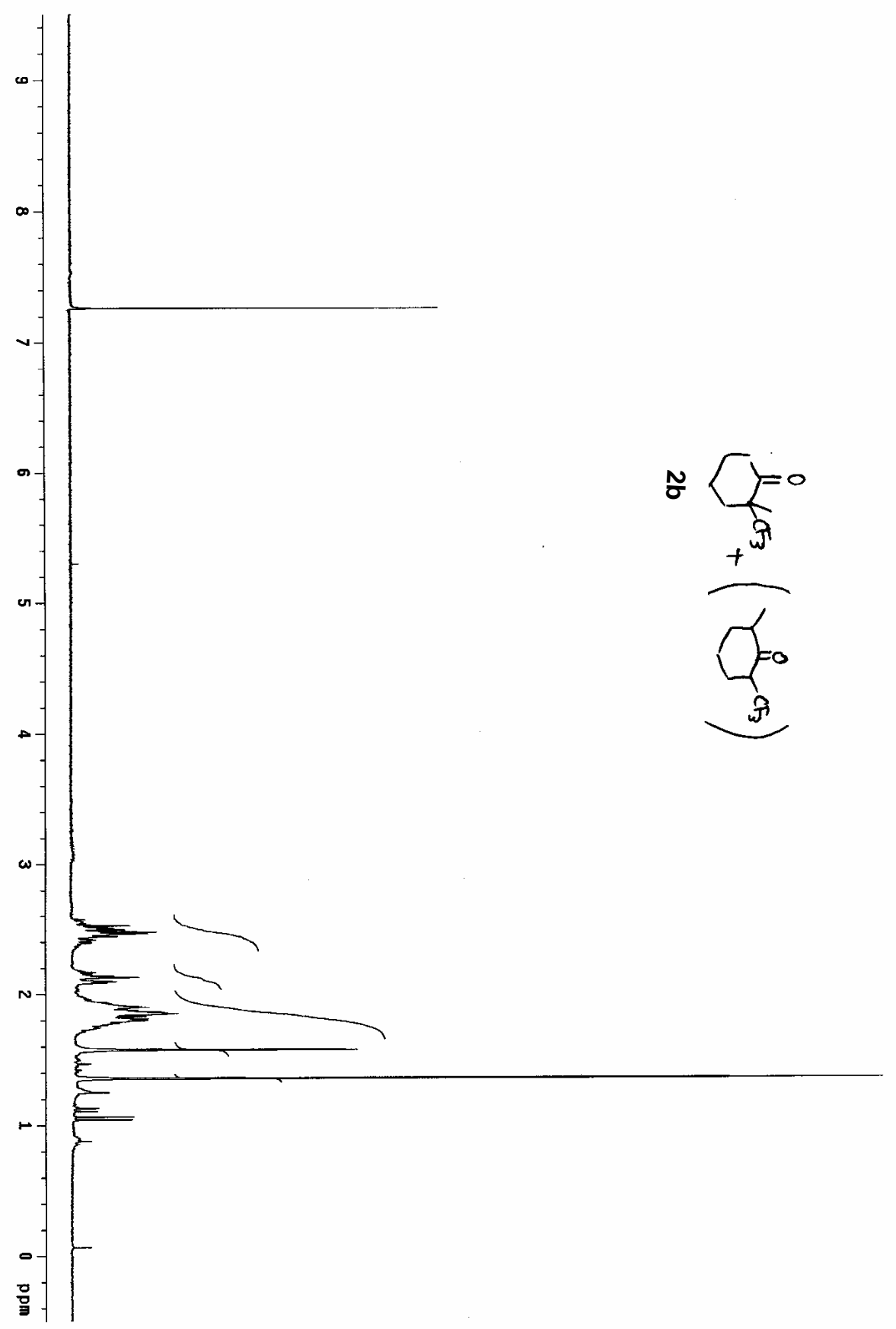




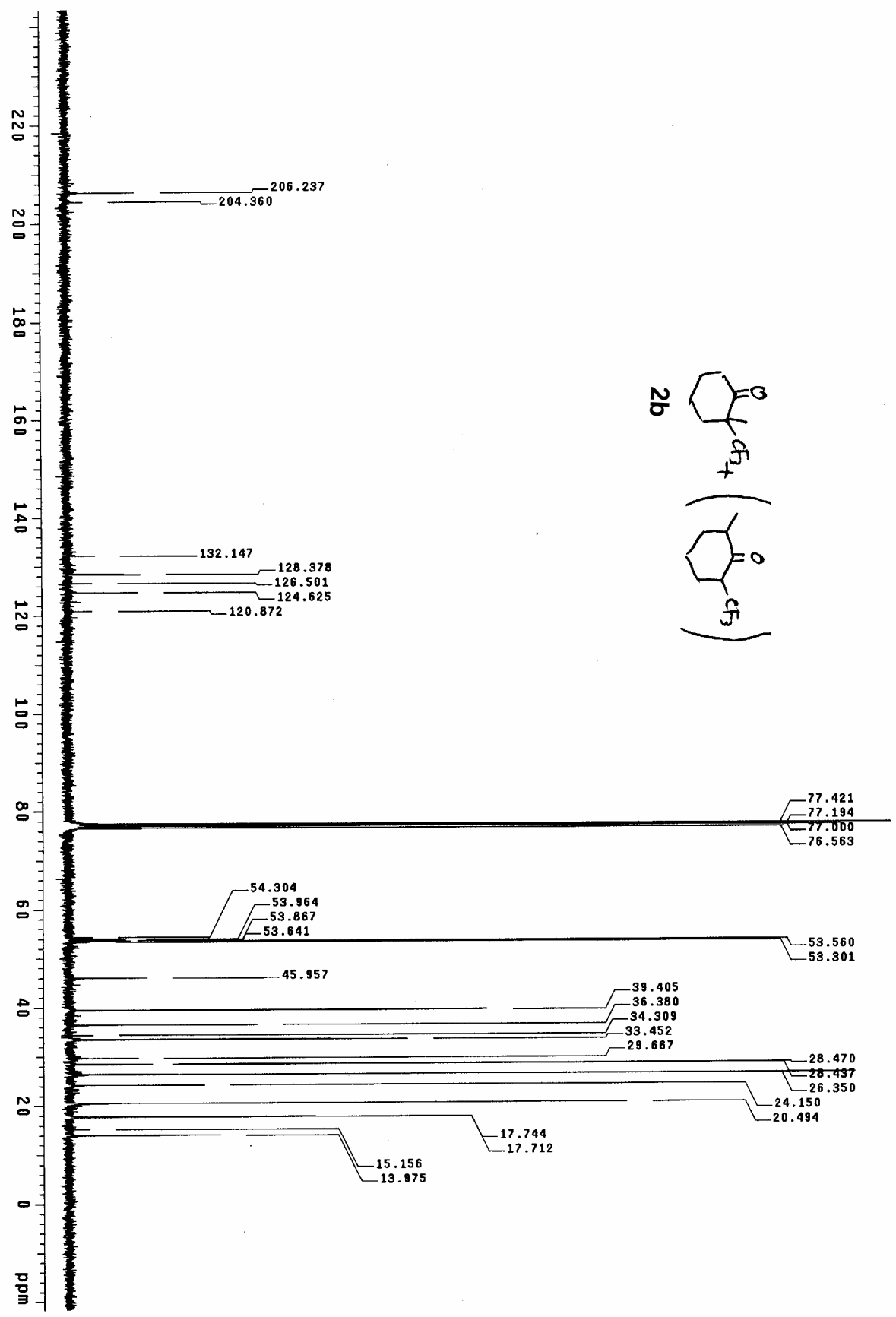




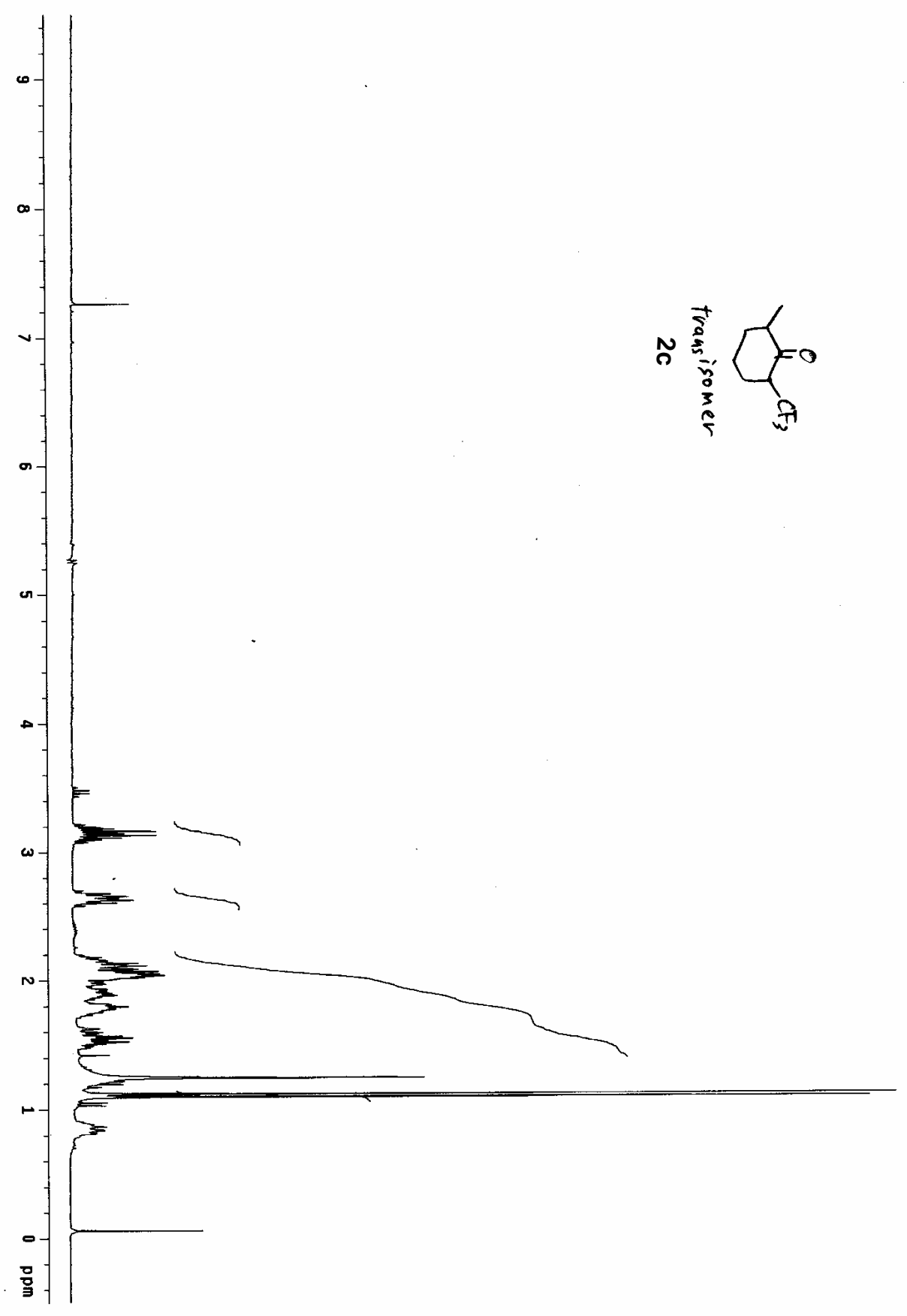




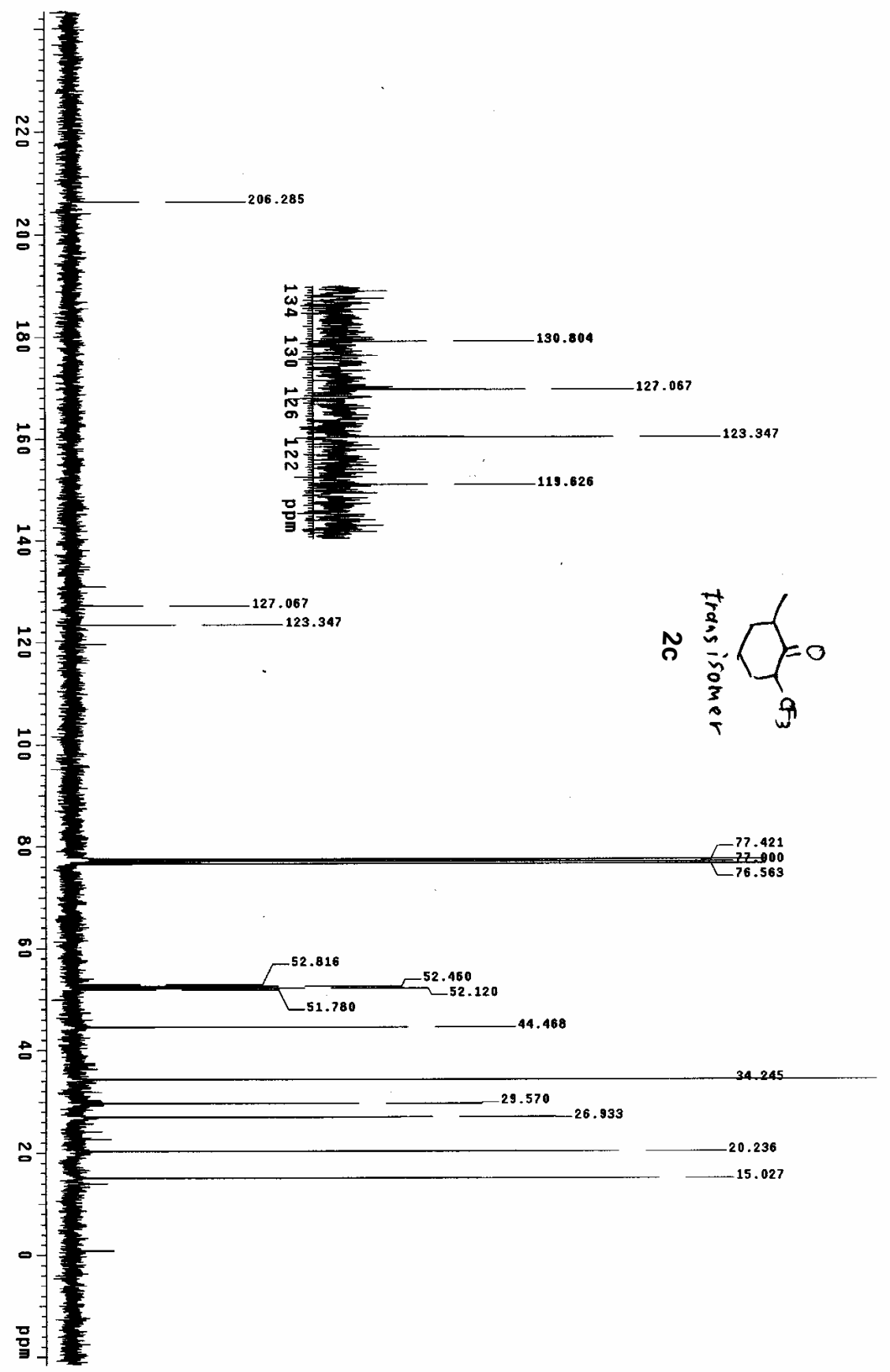




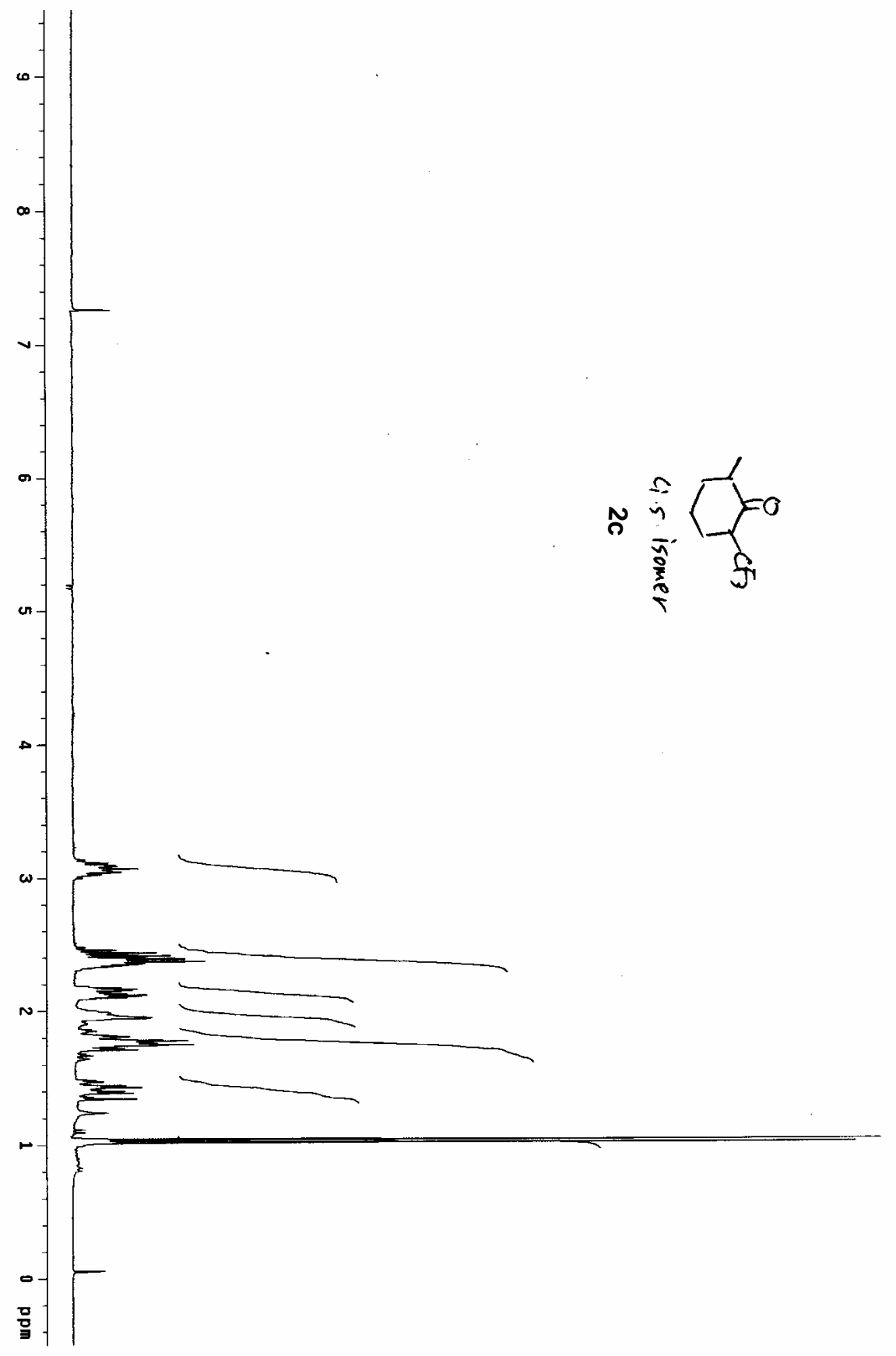




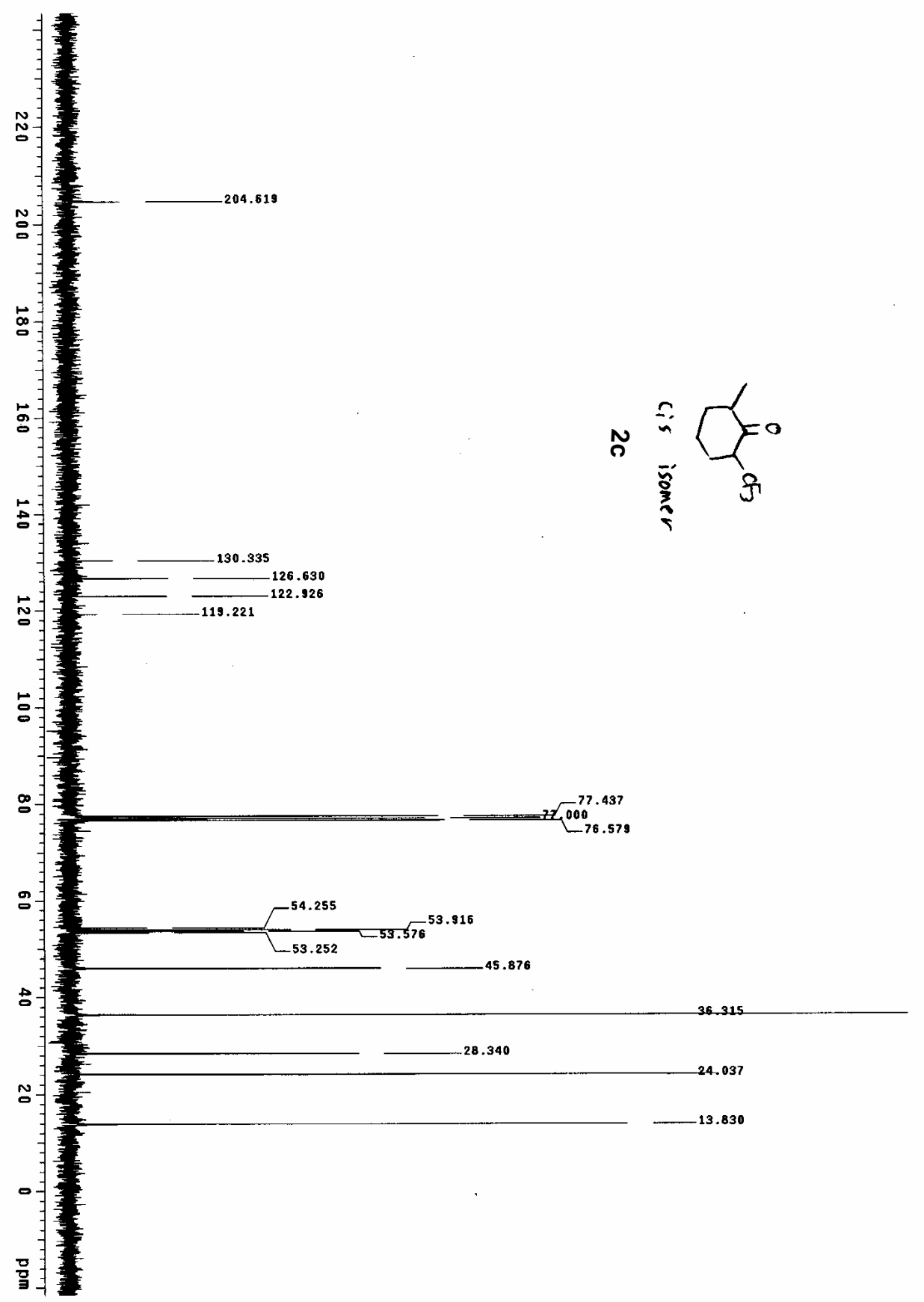




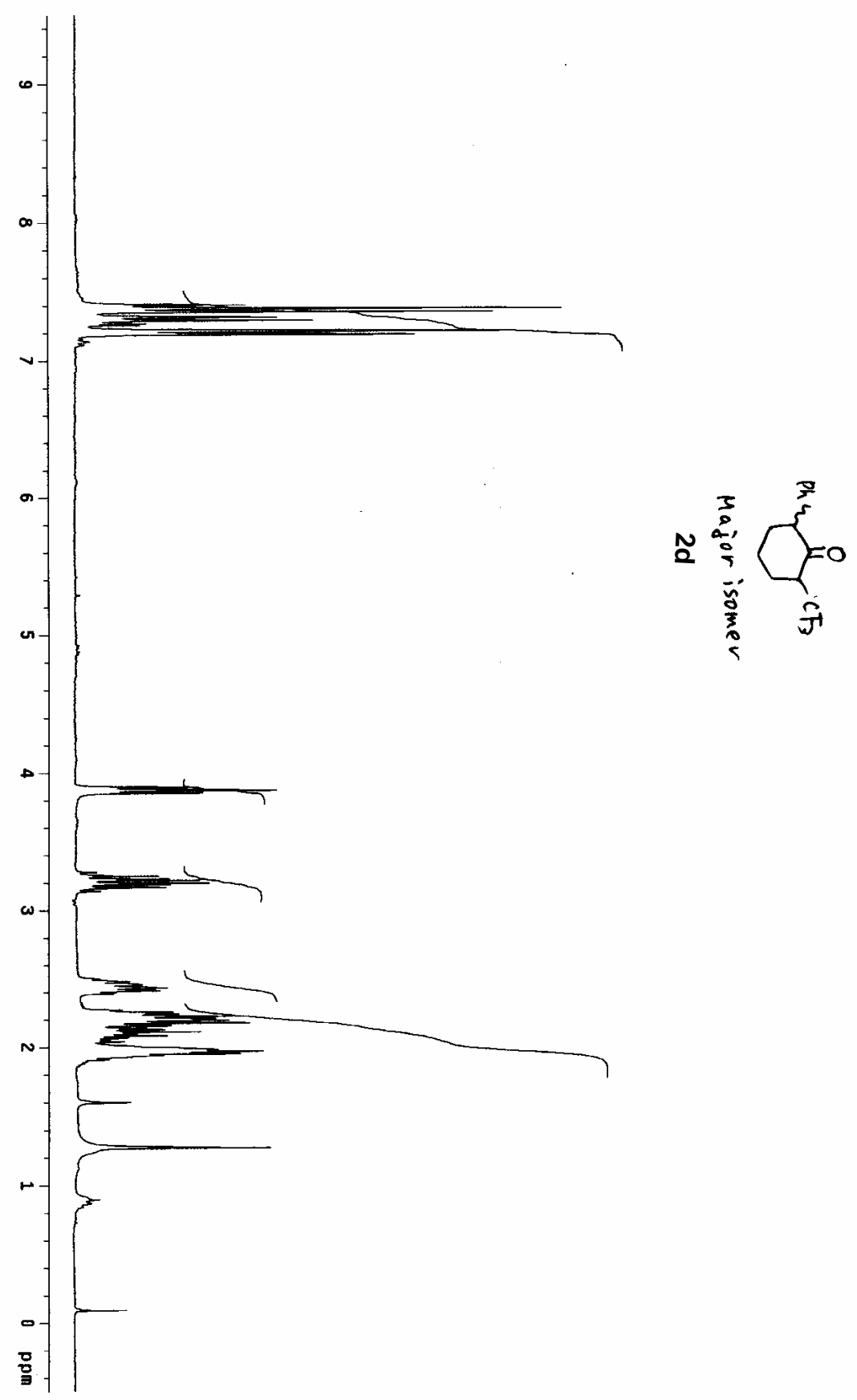




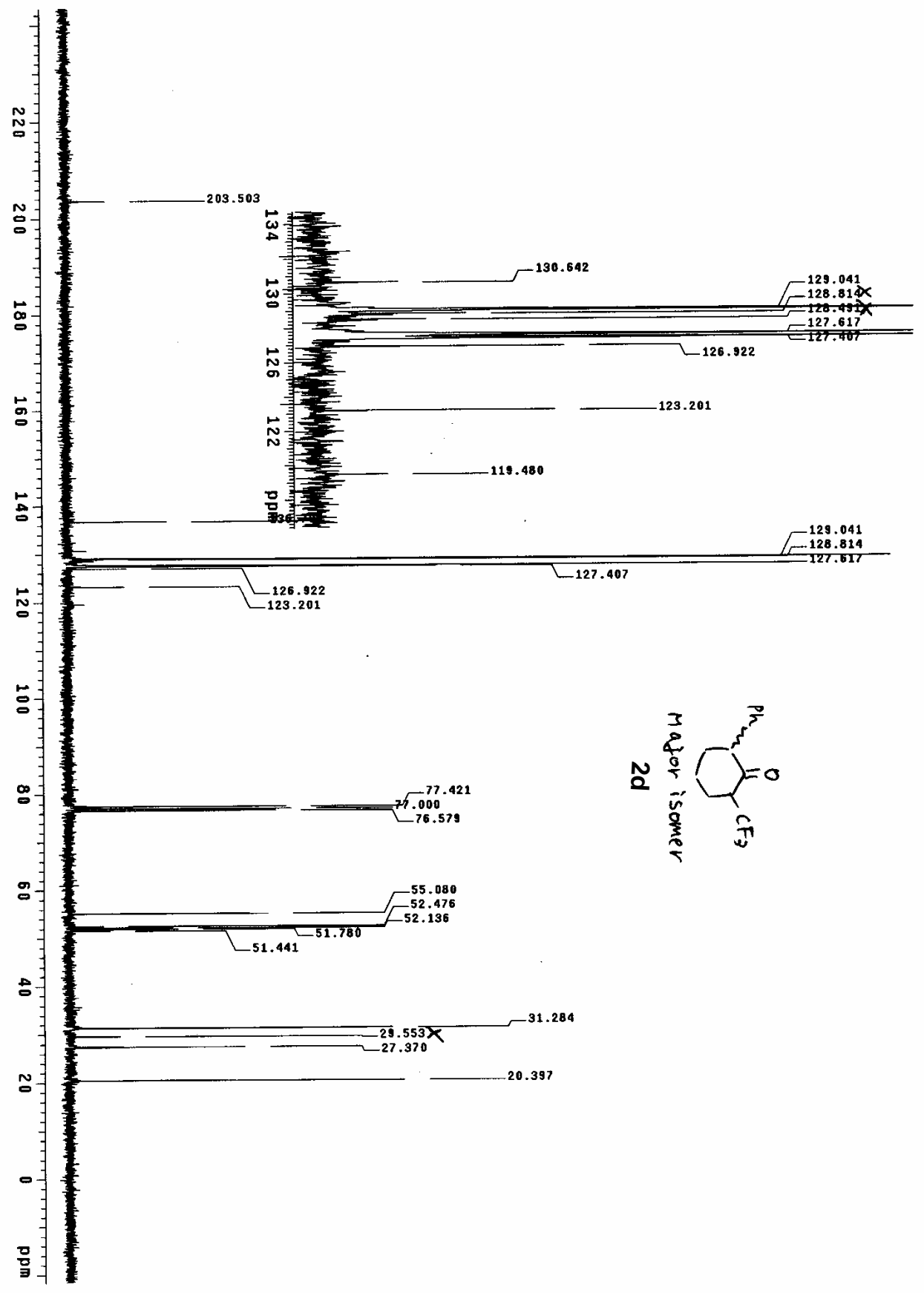




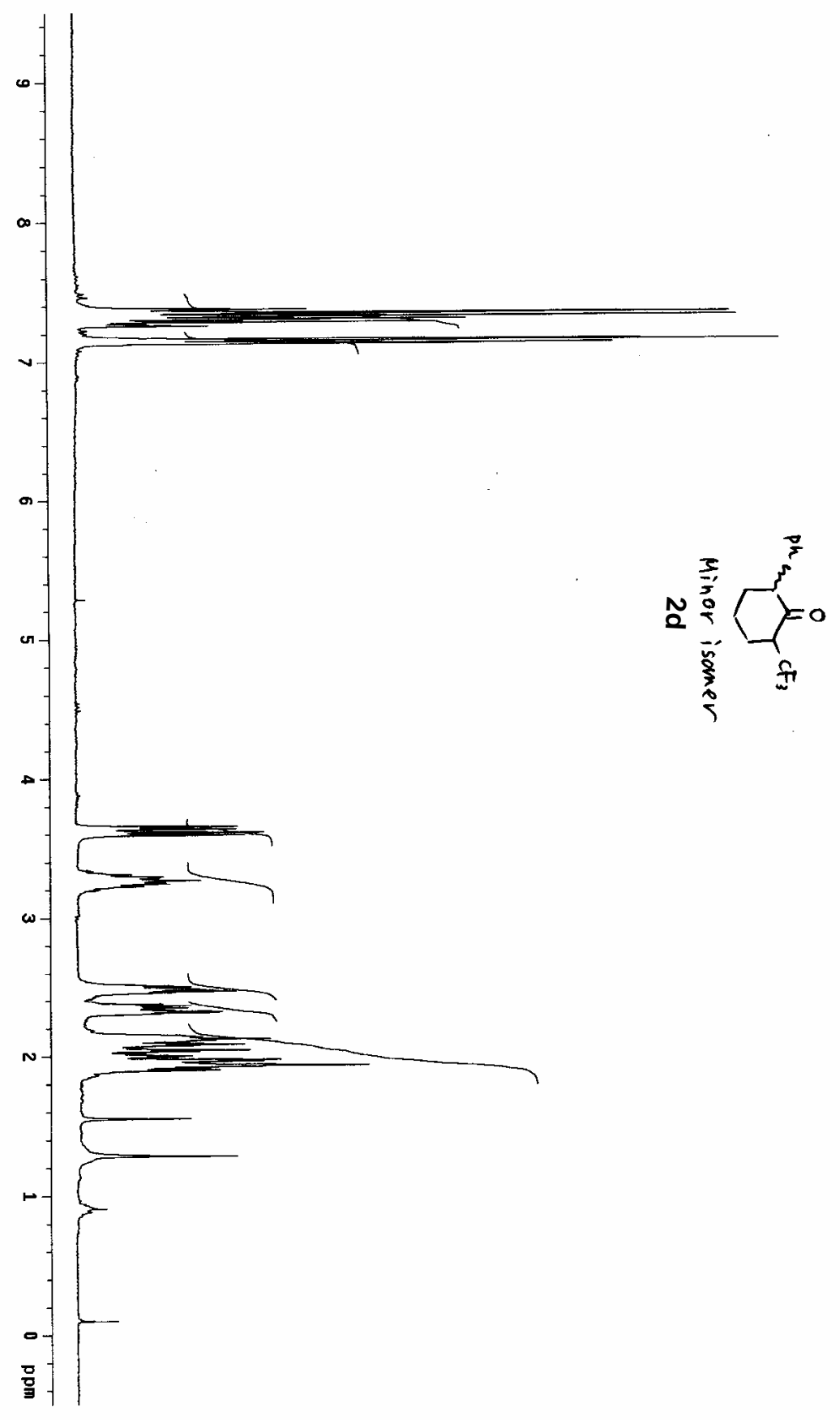




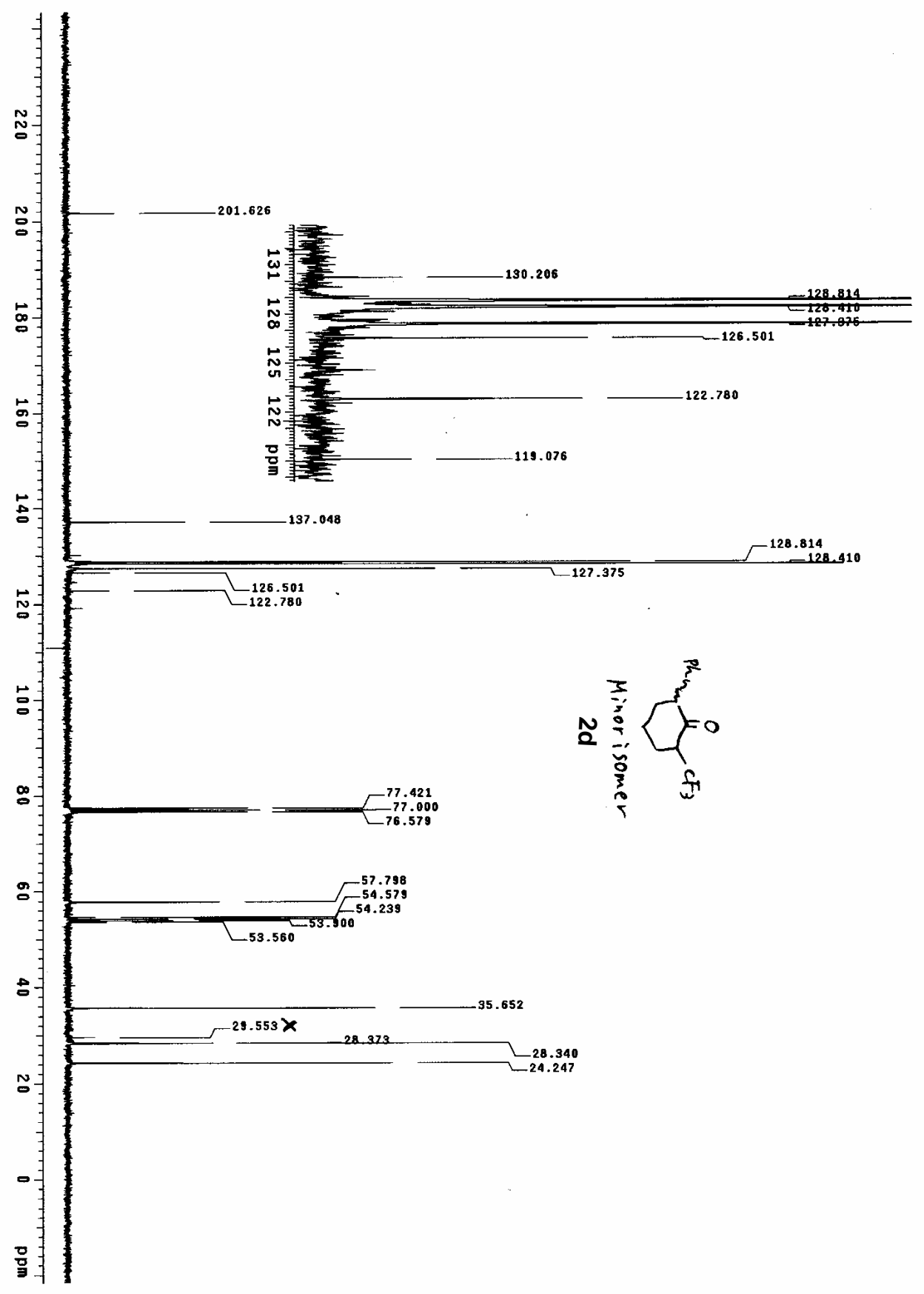




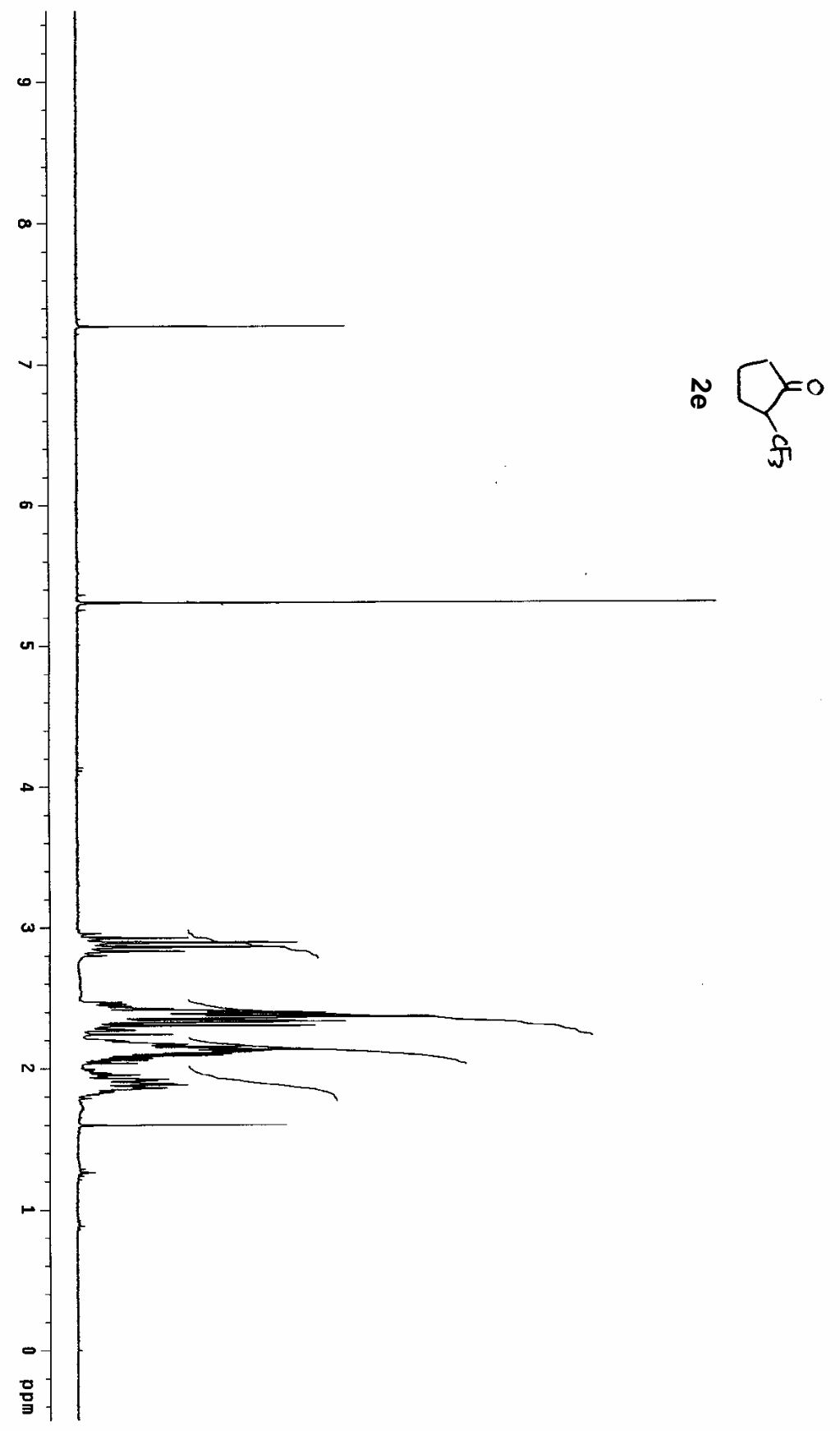




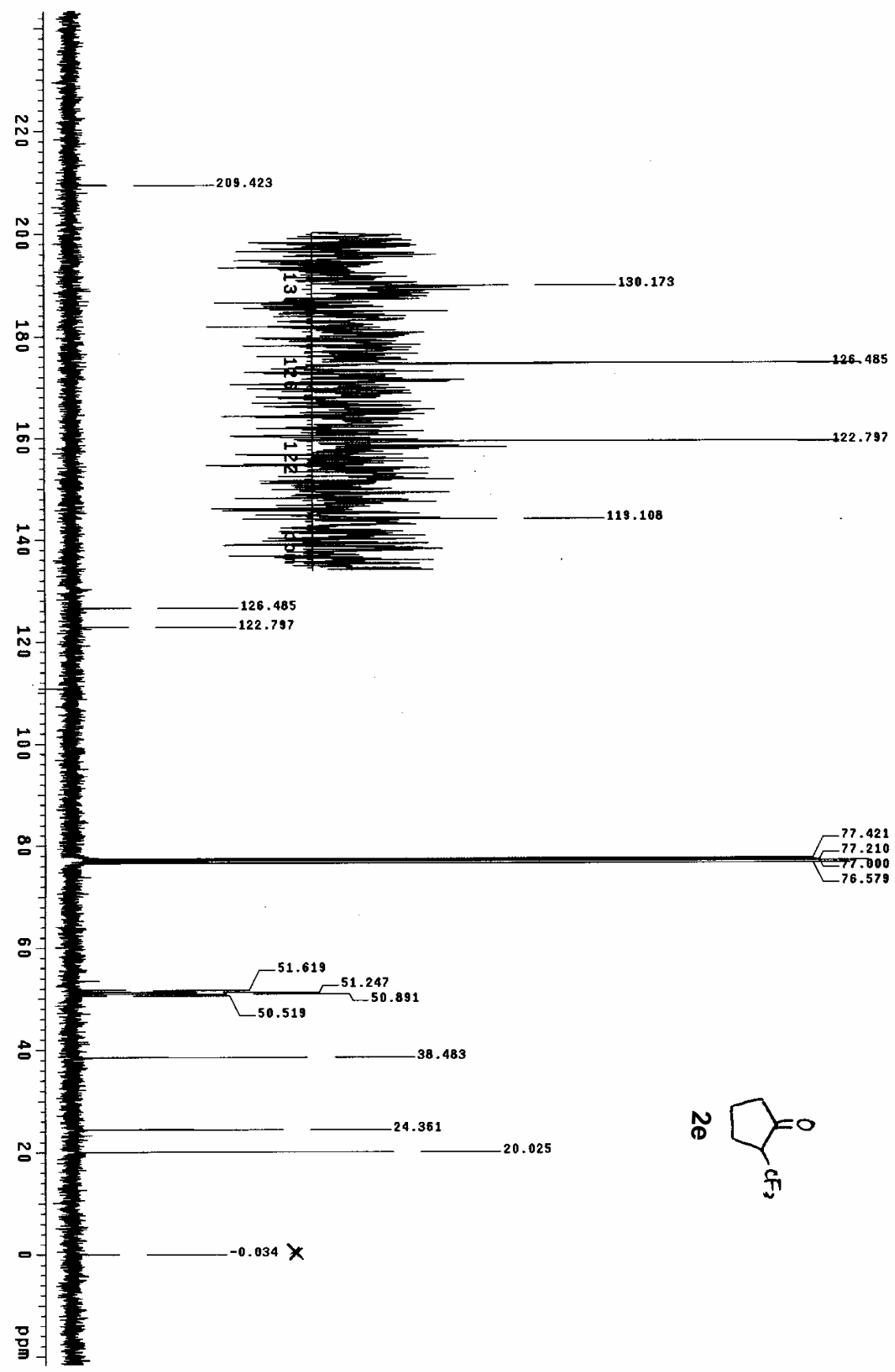




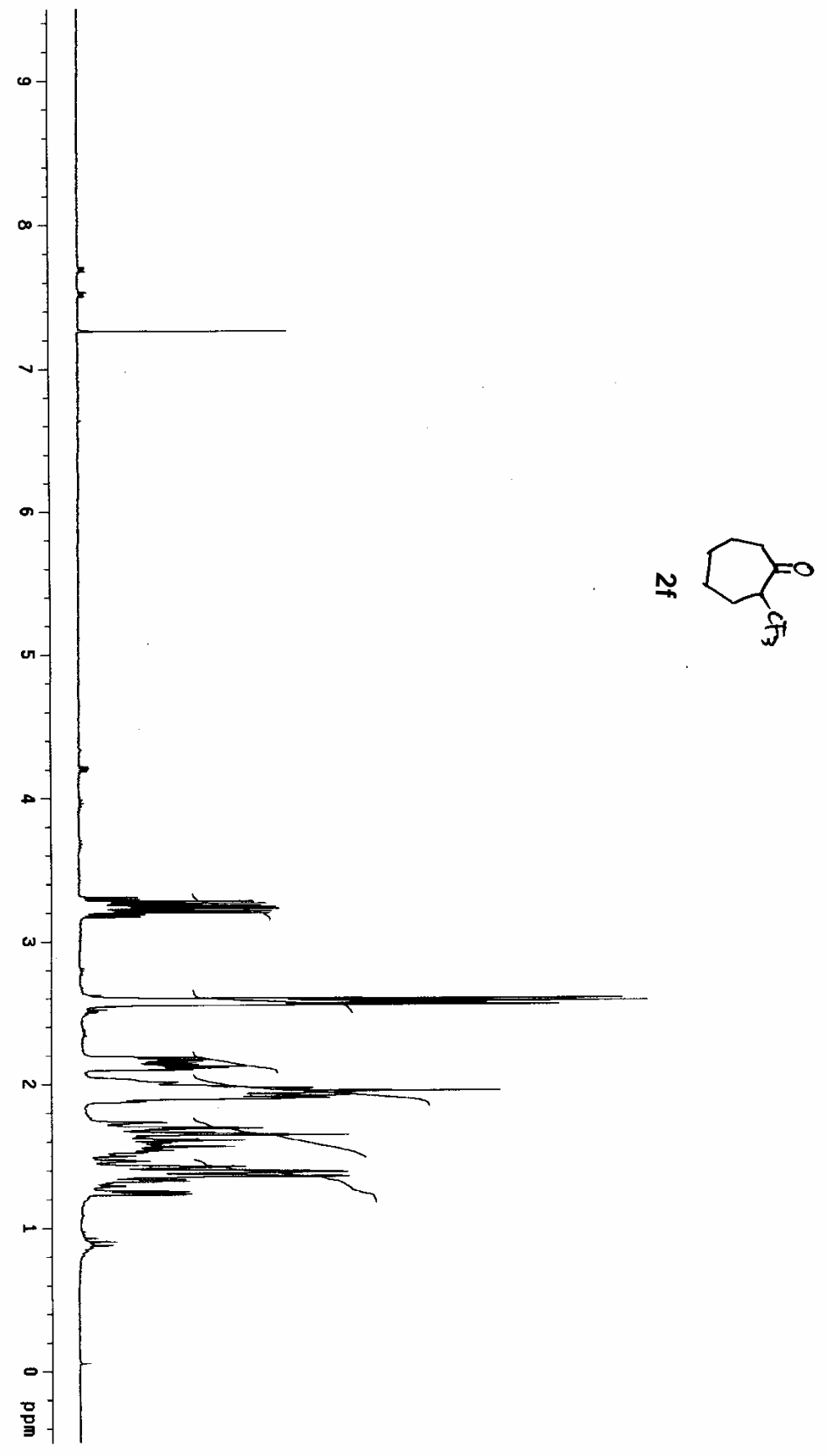




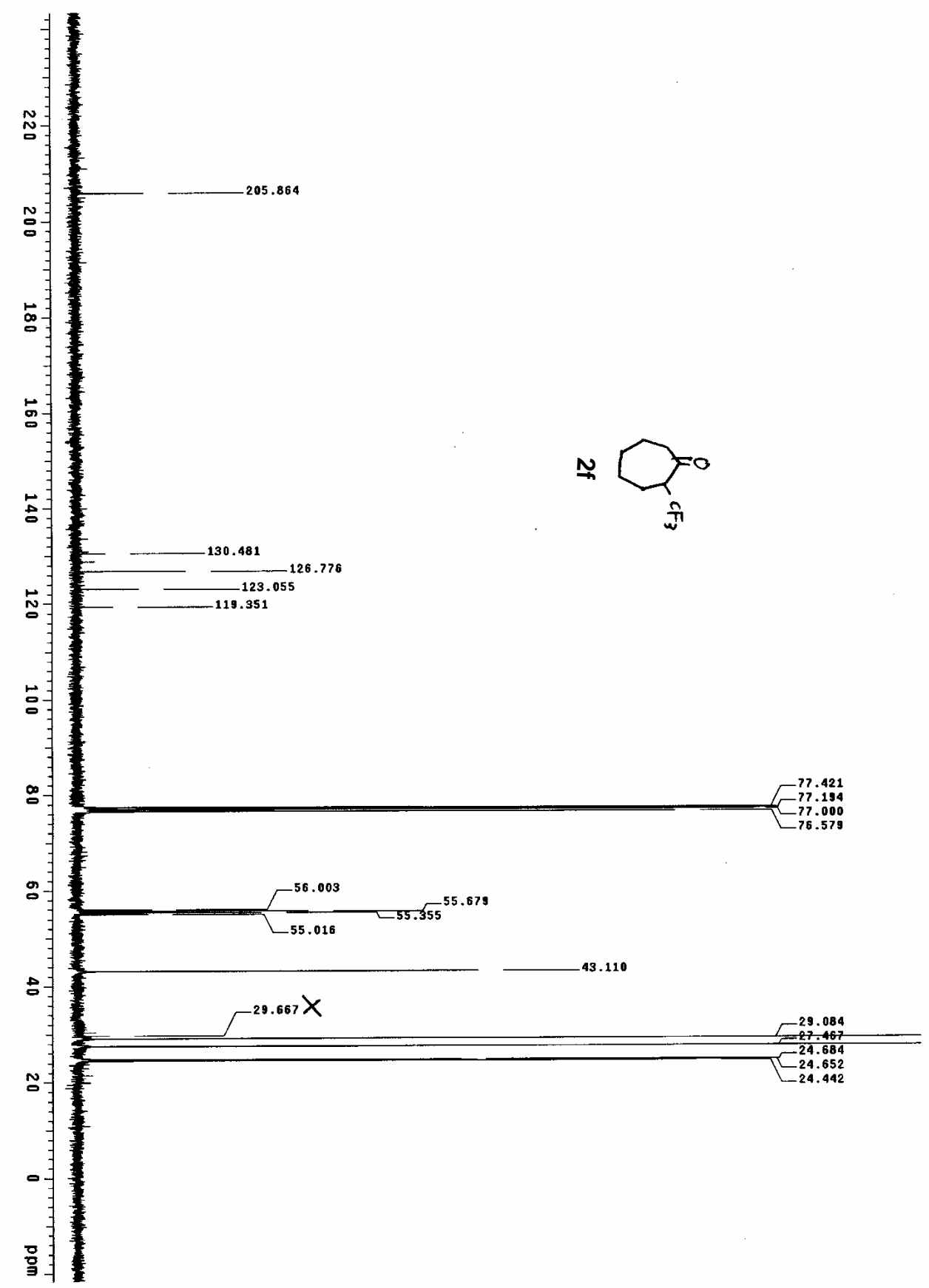




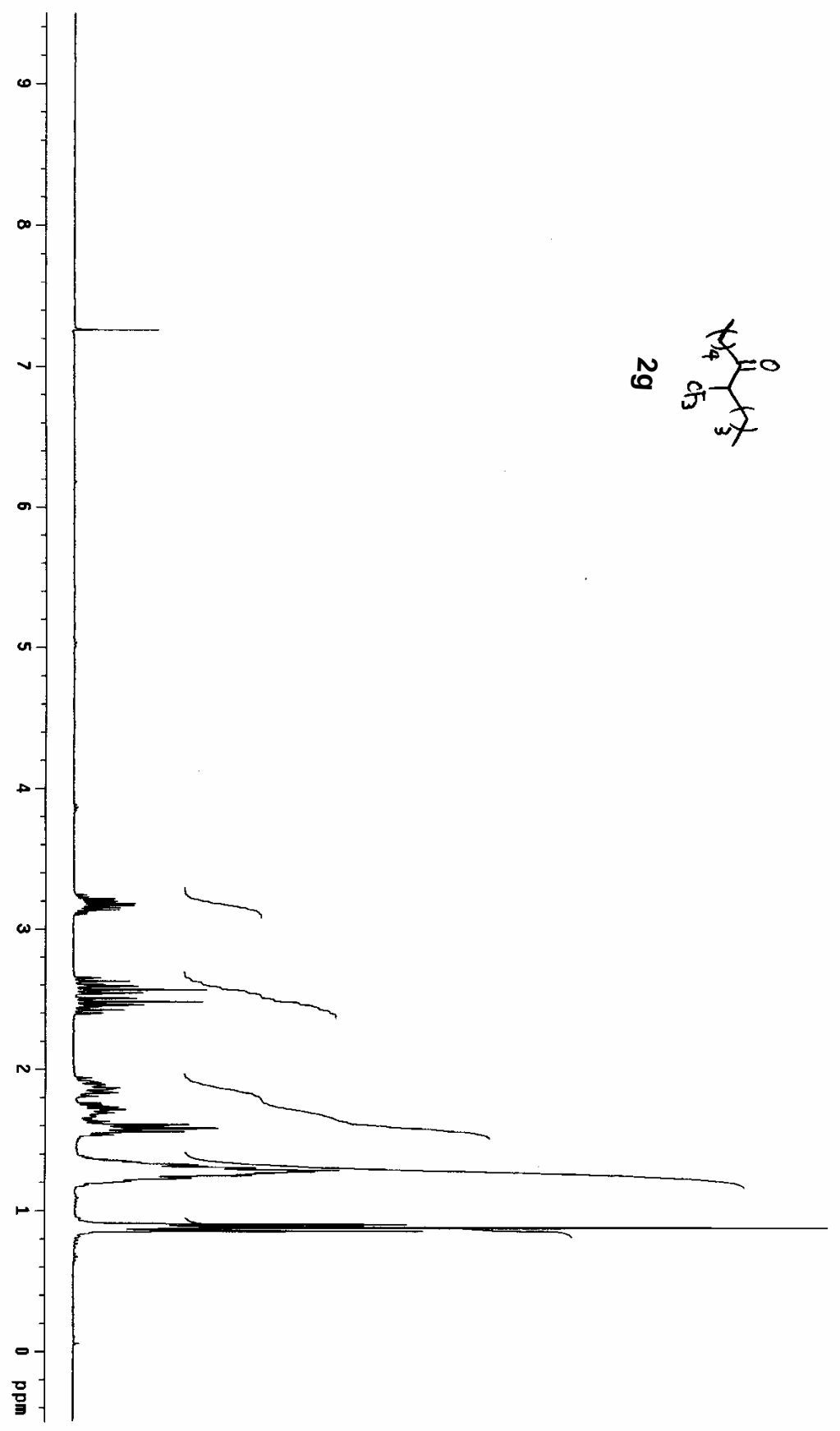




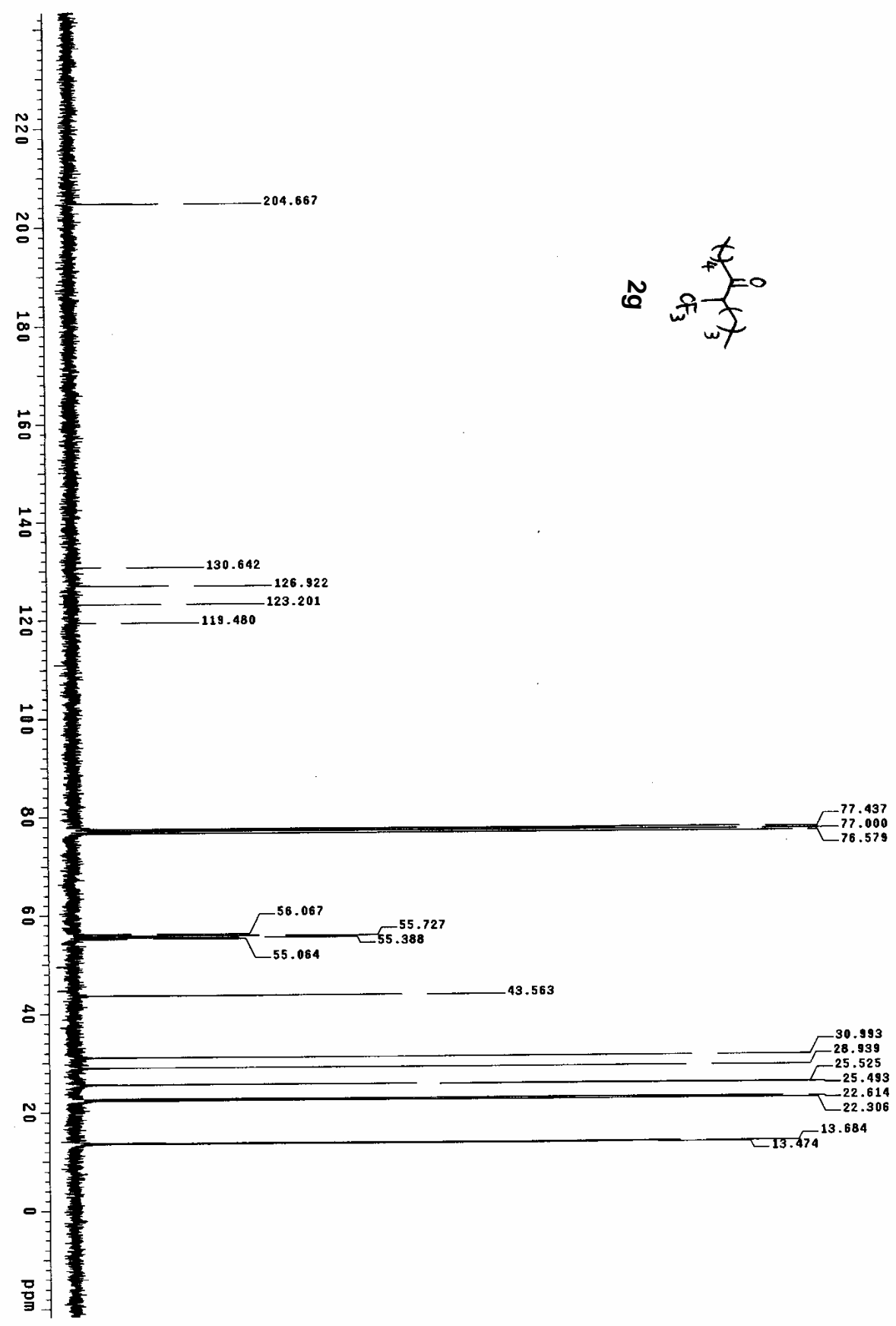




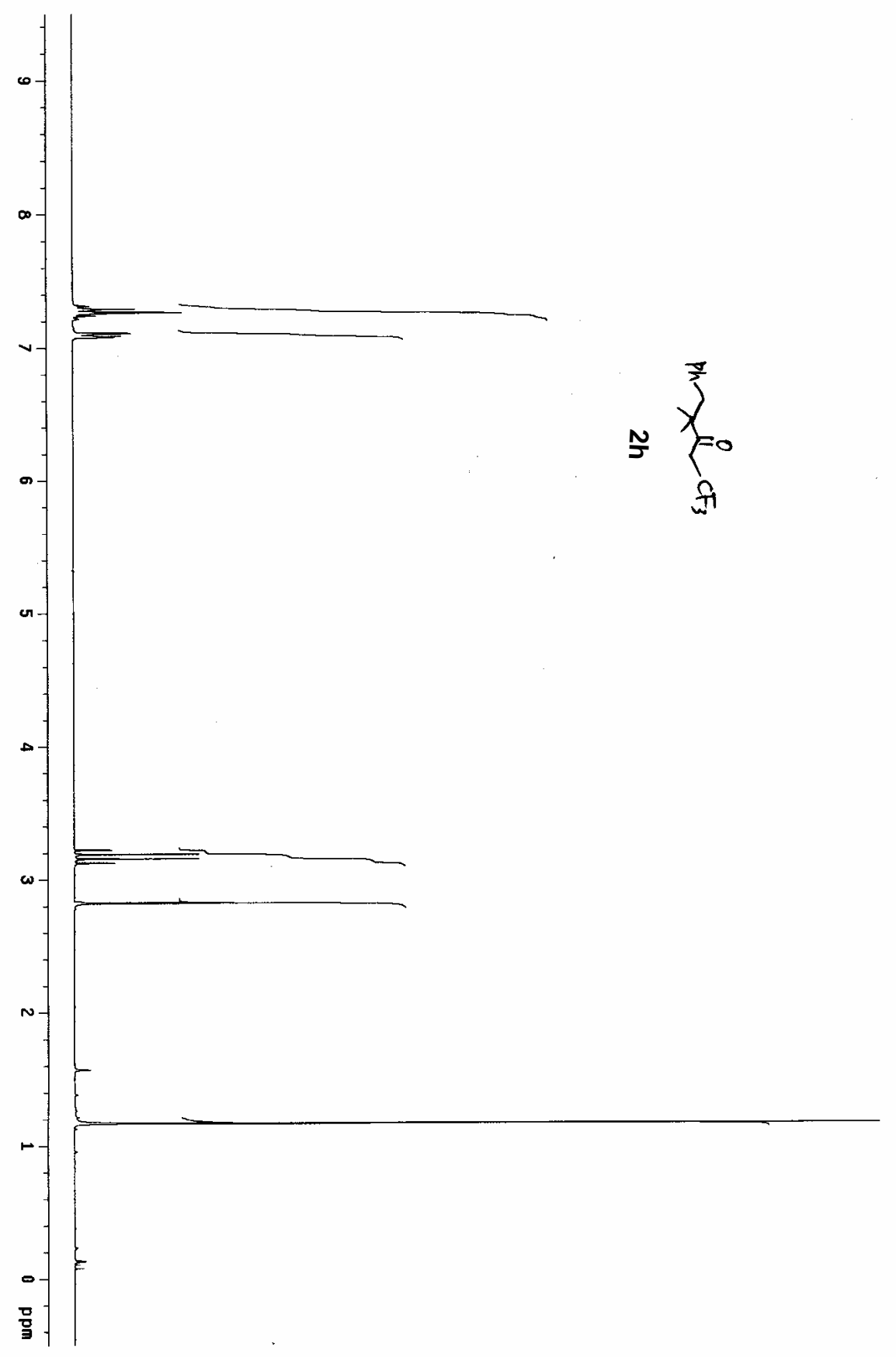




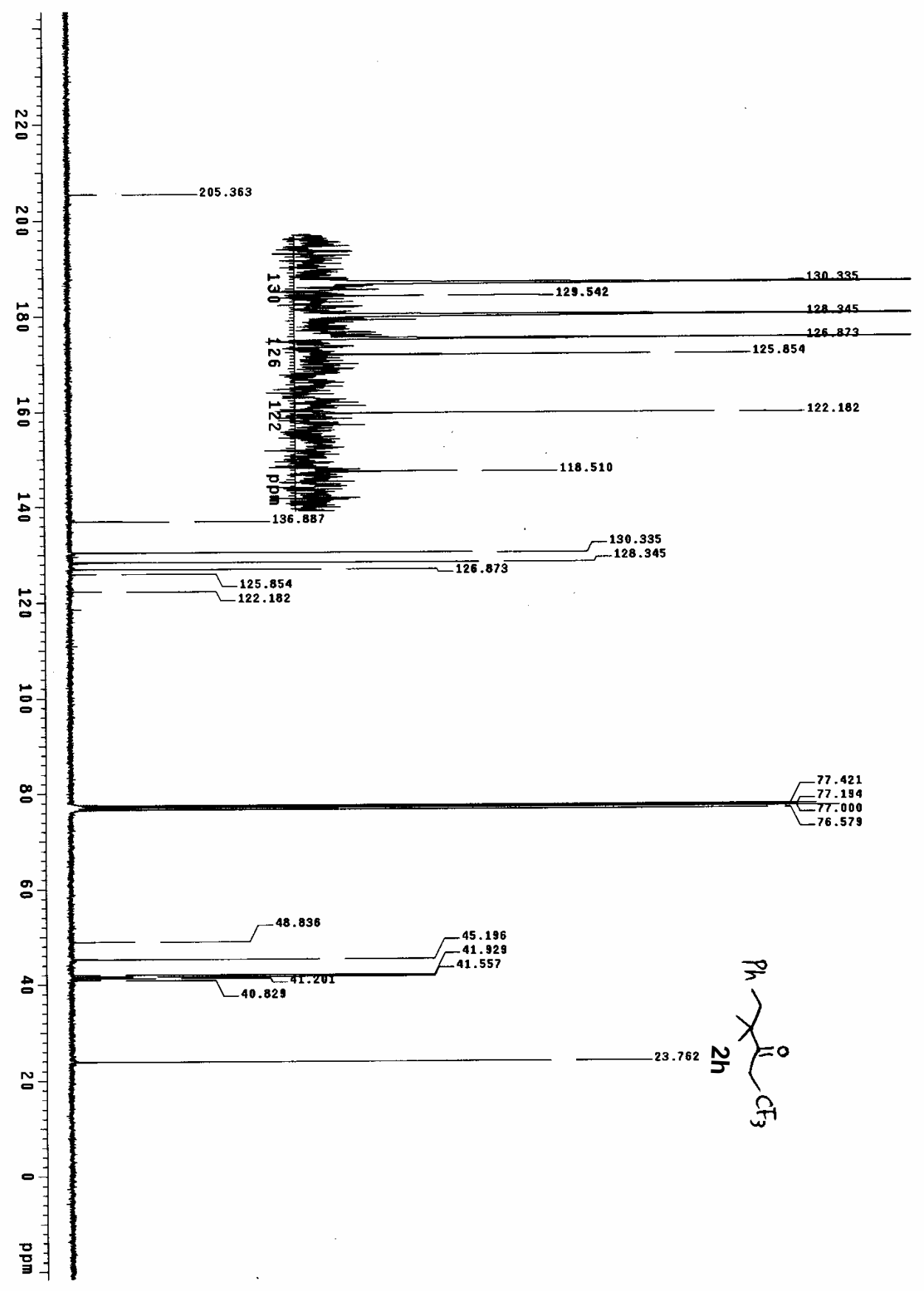

